\title{
Sensor Failure Detection and Recovery by Neural Networks
}

\section{T.-H. Guo}

Lewis Research Center

Cleveland, Ohio

and

J. Nurre

Ohio University

Athens, Ohio

Prepared for the

International Joint Conference on Neural Networks cosponsored by the International Neural Network Society and the Institute of Electrical and Electronics Engineers

Seattle, Washington, July 8-12, 1991

\section{N/SN}




\title{
SENSOR FAILURE DETECTION AND RECOVERY BY NEURAL NETWORKS
}

\author{
T.-H. Guo \\ National Aeronautics and Space Administration \\ Lewis Research Center \\ Cleveland, Ohio 44135 \\ and \\ J. Nurre* \\ Ohio University \\ Department of Electrical and Computer Engineering \\ Athens, Ohio 45701
}

\begin{abstract}
This paper describes a new method of sensor failure detection, isolation, and accommodation using a neural network approach. In a propulsion system such as the Space Shuttle Main Engine, the dynamics are usually very complicated and sometimes not well known. However, the number of variables measured is usually much higher than the order of the system. This built-in redundancy of the sensors can be utilized to detect and correct sensor failure problems. The goal of the proposed scheme is to train a neural network to identify the sensor whose measurement is not consistent with other sensor outputs. Another neural network is trained to recover the value of critical variables when their measurements fail. Techniques for training the network with a limited amount of data are developed. The proposed scheme is tested using the simulated data of the Space Shuttle Main Engine (SSME) inflight sensor group.
\end{abstract}

\section{INTRODUCTION}

In 1980, a ground test of the Space Shuttle Main Engine (SSME) experienced an erroneous measurement of the Main Combustion Chamber pressure (Pc) [1]. Pc is used for the closed loop thrust level control as well as closed loop mixture ratio calculations. The failed sensor reading led the testing to a severely abnormal operating condition. An internal fire and subsequent explosion occurred as a result of the sensor failure. The engine was virtually destroyed. Also, during the course of the Space Shuttle program there have been numerous incidents of sensor failures which caused component damage, unnecessary shutdowns and delays of the program.

In order to improve the operational reliability it is necessary to validate the measured sensor data, isolate any failed sensor and recover the failed critical measurement. There has been an extended effort in applying analytical redundancy to the sensor failure detection and isolation in the jet engine failure diagnosis problem [2]. In general, this approach utilizes the engine model and the Kalman Filter to detect and isolate sensor failures. This technique is strongly dependent upon a reliable system model which may not always be attainable in a complex system.

This paper proposes that neural networks be trained by experimental data and leam the relationships among the redundant sensors. These networks are then used to check the validity of the sensor readings and provide an estimated value for failed sensors. This paper will first describe some of the system dynamics of the Space Shuttle Main Engine. The selection and the training algorithms of the neural networks are then presented, followed by the simulation results of the proposed approach. Finally, a discussion of the research is presented.

\section{The SSME DYNAMICS}

The Space Shuttle Main Engine under study is by far the most complicated and power intense machine among propulsion engines. A simplified description of the system operation follows $[3,4]$. There are three main engines in a space shuttle orbiter. Each engine produces a sea level thrust of $375,000 \mathrm{lb}$ and a vacuum thrust of $470,000 \mathrm{lb}$. A schematic diagram of the propellant flows is shown in Fig. 1. Pressurized fuel, provided by the fuel tank, flowing through the low pressure fuel pump and the high pressure fuel pump, is fed to the regenerative cooling and the preburners. A pressurized oxidizer tank provides the oxidizer which flows through the low pressure oxidizer pump and the high pressure oxidizer pump where the output flow splits into the two prebumers and the main combustion chamber as shown in Fig. 1.

The dynamics of the system operation include: (1) the performance of turbopumps; (2) the heat exchange of the cooling flows: (3) the combustion of the two preburners and the main chamber; (4) the control valve actions; and (5) the energy properties of oxygen and hydrogen in different phases. Most of these dynamic

*NASA/OAI Summer Faculty Fellow at NASA Lewis Research Center. 
properties are based on empirical data and are highly nonlinear. For example, a hydrogen energy property table has to be used to calculate the relationships among the internal specific energy, the pressure, the temperature, and the density at a given state. In order to demonstrate the complexity of the system, the dynamics of a typical hot gas turbine, which represents only a small portion of the whole SSME system, is shown here. Given upstream pressure $P_{U}$, the upstream temperature $T_{U}$, the downstream pressure $P_{D}$, the gas constant $R$, the specific heat constant $C_{P}$, the rotational speed $S$, the specific heat ratio $\gamma$, the flowrate DW and the empirically determined turbine performance map $\mathrm{f}_{\mathrm{HG}}\left({ }^{\circ}\right)$ in Figure 2, the available torque $\mathrm{T}$ and downstream temperature $T_{d}$ can be calculated by following equations [3].

$$
\begin{aligned}
& \phi=\frac{S}{\sqrt{R_{U}}} \\
& P_{R}=\left(\frac{P_{D}}{P_{U}}\right)^{\frac{\gamma-1}{\gamma}} \\
& \tau=f_{H G}\left(P_{R}, \phi\right) \\
& T=P_{U} \tau \\
& T_{D}=T_{U}-\frac{T S}{9340 C_{P} D W}
\end{aligned}
$$

From the dynamics described above it can be seen that there exist certain defined relationships among these measured variables, although these relationships may be complicated. Further analysis reveals that analytical redundancy does exist, i.e. an unknown variable can be estimated using other related variables. However, with four turbopumps and three combustors operating simultaneously, it is extremely difficult to design a Kalman Filter type estimator for any selected measurement without grossly simplifying the dynamics.

\section{SSME SENSOR GROUPS}

There are hundreds of sensors used to collect online operational data. However, only 21 of them are used for inflight control/ shutdown purposes. These sensors include: speed sensors for three of the turbopumps; a fuel flowmeter; a pressure sensor for the main combustion chamber (MCC); pressure and
SSME PROPELLANT FLOW SCHEMATIC



Figure 1. SSME Propellant Flow Schematic

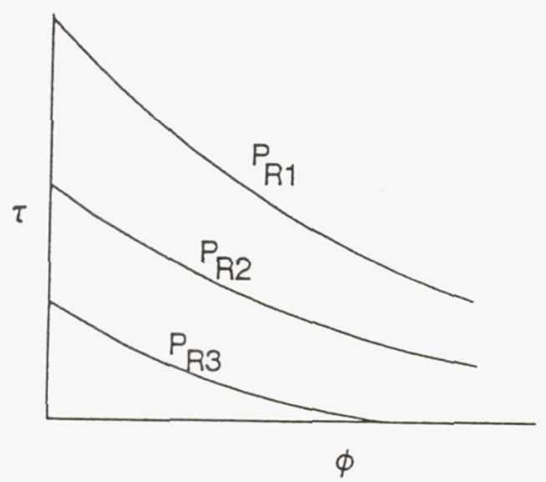

Figure 2. Hot Gas Turbine Performance Map

temperature sensors for the cooling ducts; and pressure and temperature sensors for the selected pump and turbine inlet and discharge points. Among these sensors only MCC pressure, high pressure fuel pump (HPFP) inlet flow, HPFP inlet pressure and HPFP inlet temperature are used for controlling the engine performance. The rest of the inflight sensors are used to monitor the operating condition and to activate the engine shutdown when the red-line condition is detected.

In order to simplify the problem, the scope of this study is limited to the sensor failure detection during the nominal operating condition. The study can be easily extended to detect sensor failures for abnormal operating conditions if data for these conditions is obtained. Also, only the single sensor failure problem is addressed because we assume that the simultaneous 
sensor failure situation is not likely to occur and consecutive sensor failures can be handled by cascading single sensor failures.

From the analysis of the dynamic relationships of these selected measurements, an "influence sensor map" can be constructed. This "influence sensor map" is the simplified description of how a measurement can be directly influenced by other measurements. Again, this relationship may be complicated and not intuitive even to an expert. Among the SSME sensors, there are two closely related measurement clusters, one for the fuel system and one for the oxidizer system. Figure 3 shows the "influence sensor map" for the fuel system. This cluster of measurements will be used to study the sensor failure detection and signal reconstruction using network computing. The sensors selected here are:

P6: Main Combustion Cooling Pressure

T6: Main Combustion Cooling Temperature

Qfd1: Low Pr. Fuel Pump outlet flow, in volume

Pfd1: Low Pr. Fuel Pump outlet Pressure

Tfd1: Low Pr. Fuel Pump outlet Temperature

Pfd2: High Pr. Fuel Pump exit Pressure

Tft2d: High Pr. Fuel Turbine Downstream Pressure

Sfl: $\quad$ Low Pr. Fuel Turbopump Speed

Sf2: High Pr. Fuel Turbopump Speed

Pc: $\quad$ Main Combustion Chamber Pressure

The sensor failure detection and signal reconstruction problem can be restated as:

For a given set of measurements at any time instant:

1. identify the measurement which is not consistent with others

2. estimate the value for the identified failed sensor.

\section{NEURAL NETWORK SELECTION}

The neural network structure selected for this task is a multilayer feedforward network with the sigmoidal activation function for each node (Figure 4) [5]. There are two networks to be trained for the two described functions: failure detection and lost variable estimation. The first network is to detect inconsistent sensor readings. The neural network used in this simulation consists of 10 input nodes, 30 first hidden layer nodes, 30 second hidden layer nodes, and 10 output nodes. The normalized sensor values are applied to the input nodes. The 10 output nodes on the final layer represent the confidence levels of the 10 corresponding sensor readings. The functional requirement of this network is to process a given set of normalized sensor measurements and generate a list of confidence indicators for the sensor readings. For example, for a good set of sensor readings, the output of this neural

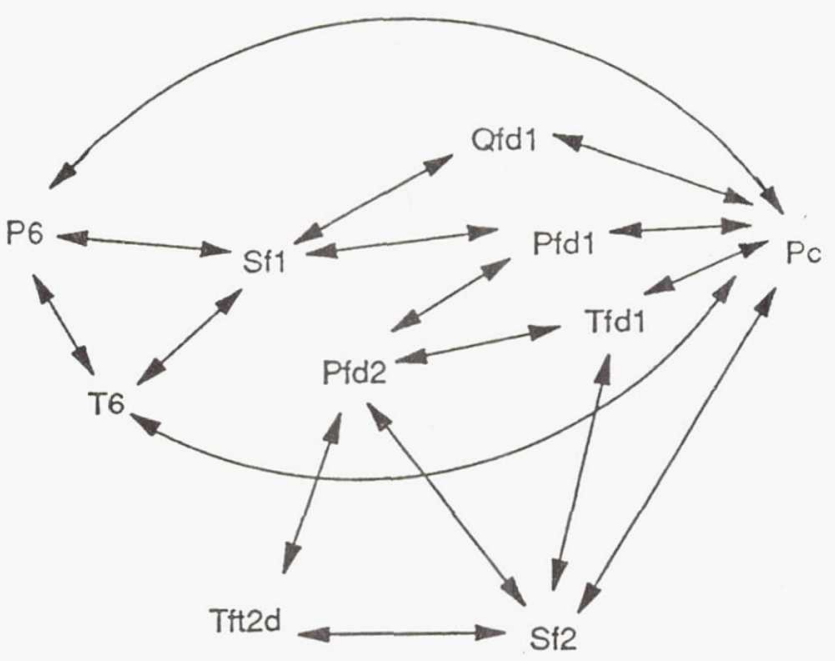

Figure 3. Influnce Sensor Map of SSME Fuel System

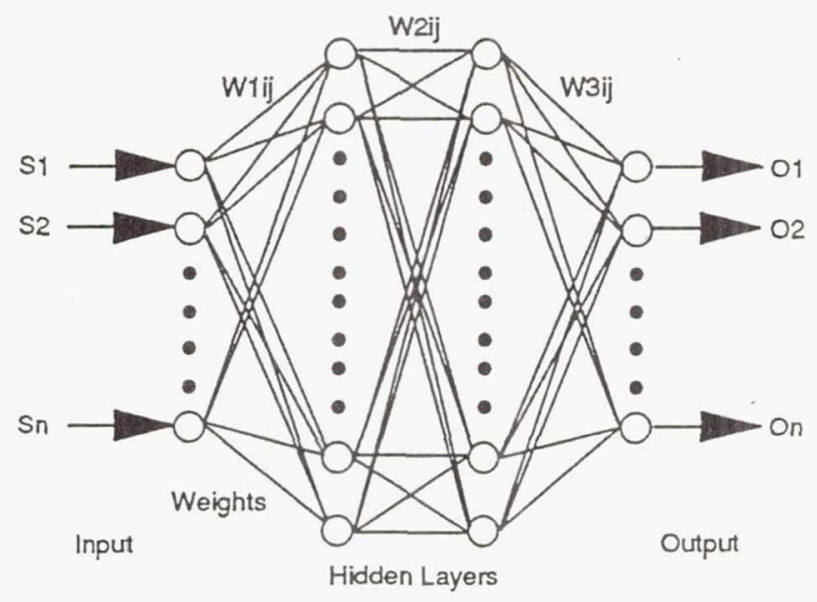

Figure 4. Feedforward Neural Network Architecture

network is expected to have high confidence indicators (values close to 1) for all sensors. If there is a sensor failure, this network's output shall be an indication of the low confidence (a value close to 0 ) in the failed sensor while indicating high confidences in other sensors.

The second neural network is to perform the recovery of the measurement due to the failed sensor. In this particular example, the network will use the other nine measurements to estimate the collapsed sensor reading identified by the previous network. The network selected here also has two hidden layers. The network chosen for the simulation consists of 9 input nodes, 30 first hidden layer nodes, 30 second hidden layer nodes, and a single output node for the sensor variable to be recovered. Usually, only the sensor readings that are used in the control loop need to be recovered. 


\section{TRAINING THE NETWORKS}

In this study, the Digital Transient Model [3] is used to simulate the dynamic behavior of the system. During start up, the main engine power level reaches 100 percent power within 4 seconds. Since this transient curve covers a very wide range of operation, it is assumed that the information gathered during this time period is rich enough to train the neural networks for both sensor failure detection and failed sensor recovery. Only data from a normal operation is used in this study. Also, the data for the first second of engine operation are discarded because most of the measurements stay constant during that time period. The data samples are recorded at the rate of $50 \mathrm{~Hz}$. In total, there are 150 sets ( 3 seconds) of sensor readings available for the neural network training.

\section{A. Training for Sensor Failure Detection}

As previously described, the purpose of this network is to single out the sensor reading which is not consistent with the other measurements. For a given set of sensor readings we can establish a range for each sensor which we consider "normal". These ranges can usually be established by combining the experts' knowledge about the process, the sensor characteristics, and the historical data base. Once the range of each measurement is selected, the goal states of the output nodes can easily be determined according to whether the measurement is within the range or not. A backpropagation algorithm is used here to train the neural network. The randomized iteration sequence is described as follows:

(1) randomly select one of the 150 sets of sensor readings,

(2) randomly select one of the 10 sensors to be trained,

(3) generate a random Gausian noise $\omega$ with zero mean and standard deviation $\sigma=1.5 \varepsilon_{\mathrm{i}}$, where $\pm \varepsilon_{\mathrm{i}}$ is the valid range for the $i$ th sensor reading $S_{i}$. Add the noise $\omega$ to $S_{i}$ to create a new sensor reading $S_{i}^{*}$. This selection of noise generates about $50 \%$ out-ofrange training samples.

(4) if $S_{i}^{*}$ is within the valid range of $S_{i}$ then set the desired output $\mathrm{O}_{i}$ of the neural network to 0.9 , otherwise set it to 0.1 , also set all other desired outputs to 0.9 ,

(5) adjust the weights according to the backpropagation algorithm,

(6) repeat steps (1) to (5) until the network can reliably indicate the failed sensor for any given situation.

\section{B. Training for Failed Sensor Recovery}

When a critical sensor reading is found to be false, it is necessary to estimate its value using other correlated measurements. A simple approach is to have one estimation network for each failed sensor that needs to be recovered. This network will have $n-1$ input nodes and 1 output node. Given the normal operation data set, the training is straight forward. The performance of the trained network is usually excellent. The training algorithm for the estimation of $i$ th sensor is:

(1) randomly select one of the 150 sets of sensor readings,

(2) apply the other 9 sensor inputs to the network,

(3) calculate the error $E_{i}=\left(S_{i}-O_{i}\right)$ for the backpropagation training,

(4) adjust the weights of the network according to the back-propagation algorithm,

(5) repeat steps (1) to (4) until the result of the estimation is acceptable.

Due to the redundancy of these selected sensors, it is expected that there is a certain degree of similarity in the estimation networks for different sensors. Thus, it may be much more efficient to have one estimation network that can estimate any selected missing variable. A single network to recover all variables in the SSME fuel system will have 10 input nodes and 10 output nodes. The training algorithm is more complicated and the performance is not as good as a single sensor estimator. Here, we limit our scope to the single estimator only.

\section{SIMULATION RESULTS}

As described in the previous section, the data generated by the Digital Transient Model (DTM) is used in the simulation. Initially, the data collected during the start up transient (i.e. $1.0 \leq$ Time $<4.0$ seconds) are used to train the neural networks. The first network is trained to capture the relationship among the measurements so that a failed sensor can be identified. Variable step size for the weight adjustments is used to help fine tune the network for better performance. Figure 5 shows the percentage of error during the training of the network. The error percentage is calculated for every 2,500 training iterations. It can be seen that the network is able to reach more than $90 \%$ accuracy in predicting any given sensor failure after about one million samples. Further fine tuning has reduced the error to less than $5 \%$. These errors occur in the neighborhood of the defined cutoff values of valid sensor readings. Because of the continuous nature of the selected network it is reasonable to have a gray area which indicates that the sensor failure is "uncertain". A second network which is to recover the Main Combustion Chamber Pressure measurement is also trained using the start up transient 
data. The input to this network consists of the signals from the other 9 sensors and its only output is the estimation of MCC pressure. The training is straightforward and the estimation results are within a few percent after several thousand iterations.

These two networks were tested for an extended run of the DTM simulation. In this simulation, the controller starts the engine, powers the engine to $100 \%$ in four seconds, holds at $100 \%$ for one second, reduces the engine power to $65 \%$ in the next five seconds, holds at $65 \%$ for three more seconds, and finally gradually increases the power to $100 \%$. This is to emulate the operation profile of the SSME during the so-called "Max-Q Throttle" operation.

\section{Case 1: HPFTP Speed Sensor Failure at $T=7.0$}

Figure 6 shows the case that the High Pressure Fuel Turbopump speed sensor $\mathrm{Sf} 2$ starts failing at $\mathrm{T}=7.0$. The failure is a soft failure, i.e. a degraded reading. The rate of failure is -350 RPM per second off the actual value. It can be seen that the confidence checking network is able to detect the discrepancy within 0.5 seconds by indicating the confidence of that sensor is low (close to 0 ). Figure 7 shows the outputs of the network during the $\mathrm{Sf} 2$ sensor failure. It shows that the failed sensor can be clearly identified within a very short period of time after it started degrading.

\section{Case 2: MCC Pressure Sensor Failure at $T=8.0$}

Figures 8 and 9 show the case in which the Main Combustion Chamber pressure sensor Pc starts failing at $\mathrm{T}=8.0$. The rate of failure is -300 PSI per second off the actual value. Figure 8 shows the outputs of the network which clearly indicate high confidence on all other sensors while singling out the Pc sensor failure. Figure 9 indicates that the confidence level of the measurement falls quickly from high (close to 1 ) to low (close to 0 ) when the measured value moves away from the real value. The on-line estimation of Pc using the second network is also shown in Figure 9. The estimated value of Pc closely follows the actual value and can be used for backup when the Pc sensor fails. This arrangement provides an uninterrupted and undegraded control after the sensor failure.

\section{CONCLUSIONS}

Neural networks are proposed to detect sensor failures and recover the lost measurements from a group of redundant sensors. A two step approach is employed. The first network is trained to detect the sensor which is inconsistent with other sensor readings. The second network is trained to recover the sensor readings which are critical in operation. This approach is especially useful when the relationship among these

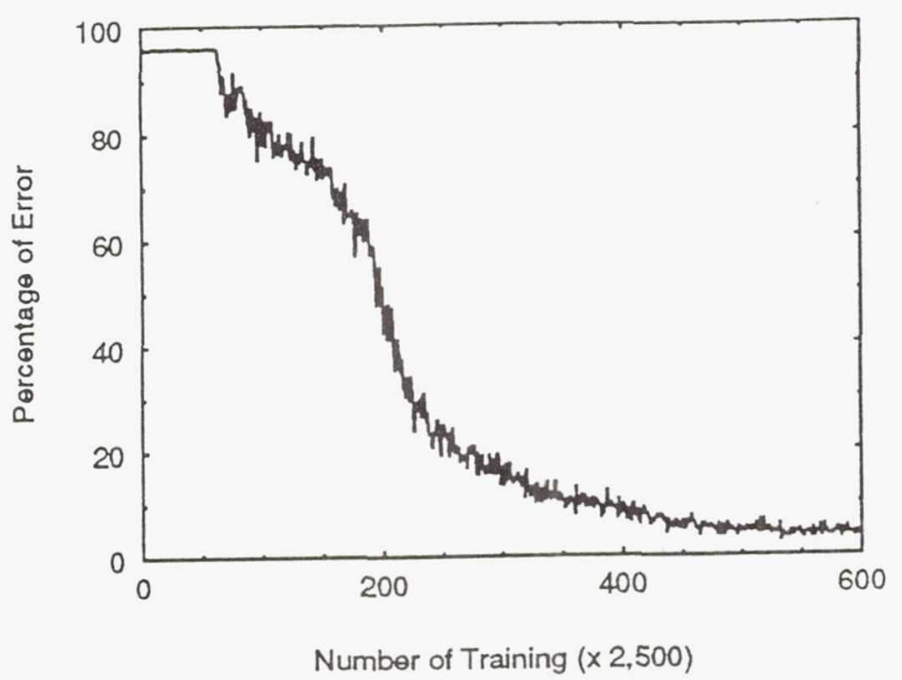

Figure 5. Error vs. Training Iterations

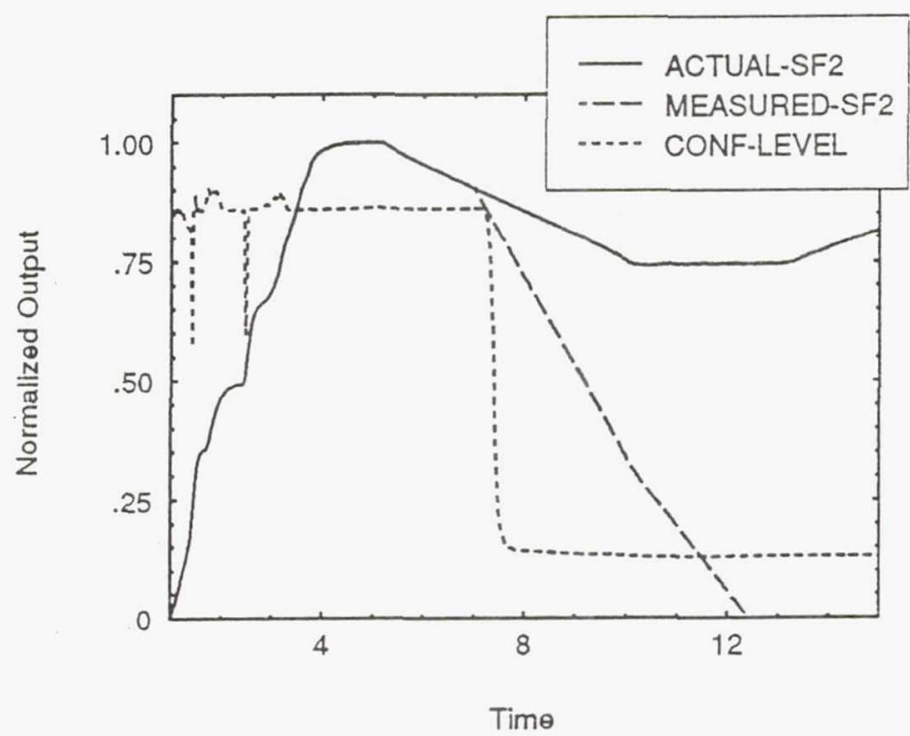

Figure 6. Sf2 Sensor Degraded at $T=7.0$ Seconds

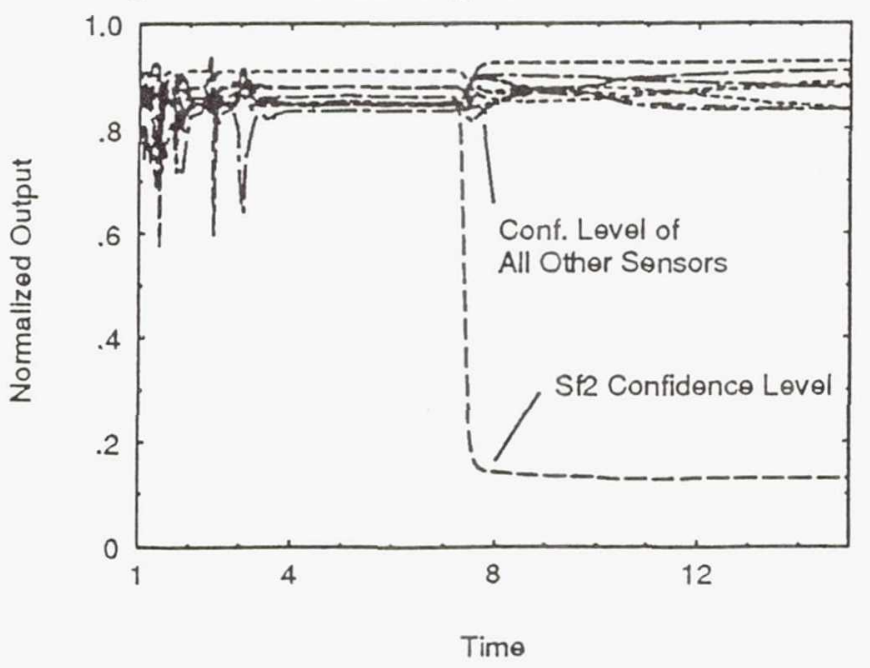

Figure 7. Outputs of the Conf. Checking Net in Case 1 
sensors can not be clearly identified or is too complex for a Kalman Filter estimator. The network can be trained using the experimental data for the selected condition. The dynamic relationship among the sensors is leamed using the back-propagation algorithm.

The proposed approach is applied to the Space Shuttle Main Engine inflight sensor group through the Digital Transient Model Simulation. The results clearly show the adequacy of the approach under the tested condition. It is conceivable that the approach can be extended to cover other operating conditions if the sensor data for those conditions are collected and applied to training.

The high speed capability of neural networks makes the proposed approach even more attractive in the realtime control problem [6]. It was shown in this study that the sensor measurements used for control purposes can be easily recovered without delay. This feature is especially useful in the design of an Intelligent Controller where real-time diagnostics and accommodation is one of the key issues.

\section{REFERENCES}

[1] H. A. Cikanek, "Characteristics of Space Shuttle Main Engine Failures," AIAA Joint Propulsion Conference, June 29 - July 2, San Diego, CA.

[2] W. C. Merrill, J. C. DeLaat, and W. M. Bruton, "Advanced Detection, Isolation, and Accommodation of Sensor Failures: Real-Time Evaluation," AIAA J. Guidance, Control Dyn., 11, No. 6, 1988.

[3] Rockwell International Corp., "Engine Balance and Dynamic Model," Report FSCM No. 02602, Spec. No. RL00001, 1981.

[4] Rockwell International Corp., "SSME Engine, Redline and Control Overview," Rep. BD 88-62, 1988.

[5] R. P. Lippmann, "An Introduction to Computing with Neural Nets", IEEE ASSP Magazine, April 1987.

[6] W. C. Merrill and C. F. Lorenzo, "A Reusable Rocket Engine Intelligent Control," NASA TM 100963, AIAA-88-3114, July 1988.

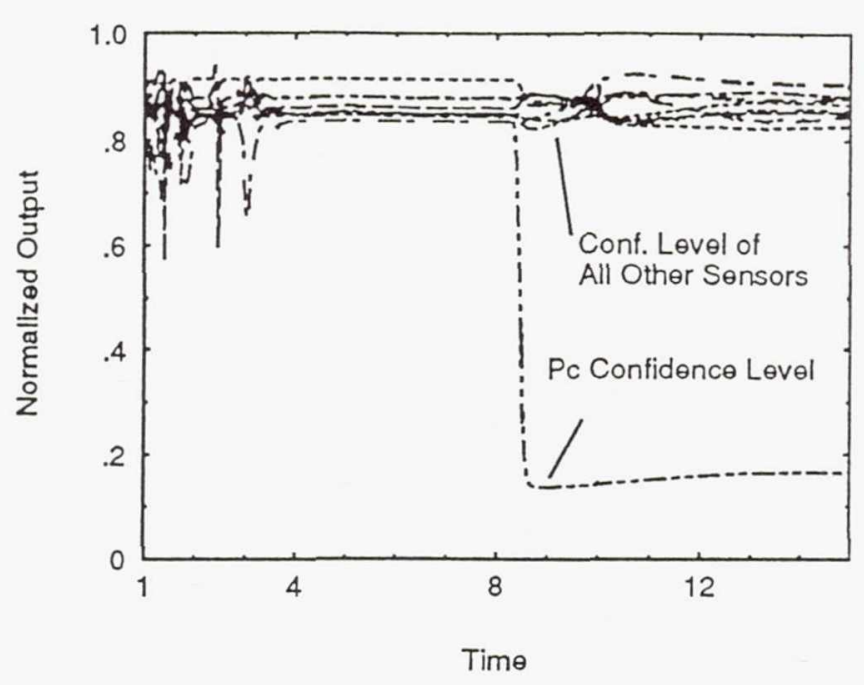

Figure 8. Outputs of the Conf. Checking Net in Case 2

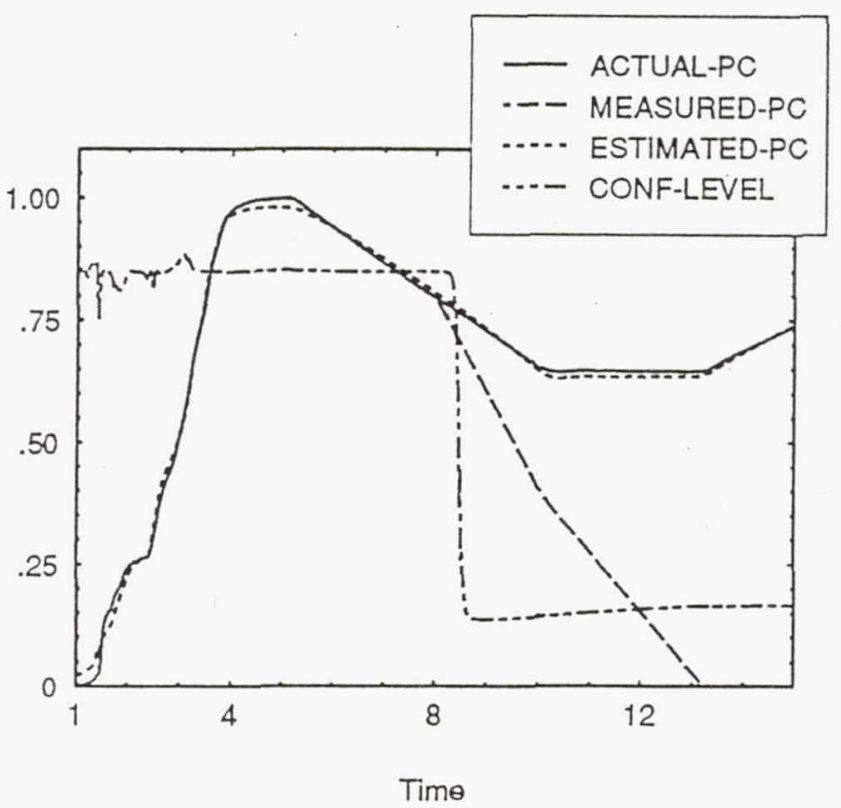

Figure 9. Pc Sensor failure at $\mathrm{T}=8.0$ Seconds 


\begin{tabular}{|c|c|c|c|}
\hline \multicolumn{4}{|c|}{ Report Documentation Page } \\
\hline $\begin{array}{l}\text { 1. Report No. } \\
\text { NASA TM-104484 }\end{array}$ & 2. Government Accession No. & \multicolumn{2}{|c|}{ 3. Recipient's Catalog No. } \\
\hline \multirow{2}{*}{\multicolumn{2}{|c|}{$\begin{array}{l}\text { 4. Title and Subtitle } \\
\text { Sensor Failure Detection and Recovery by Neural Networks }\end{array}$}} & \multirow{2}{*}{\multicolumn{2}{|c|}{ 5. Report Date }} \\
\hline & & & \\
\hline & & \multicolumn{2}{|c|}{ 6. Performing Organization Code } \\
\hline \multirow{3}{*}{$\begin{array}{l}\text { 7. Author(s) } \\
\text { T.-H. Guo and J. Nurre }\end{array}$} & & \multirow{2}{*}{\multicolumn{2}{|c|}{$\begin{array}{l}\text { 8. Performing Organization Report No. } \\
\text { E-6330 }\end{array}$}} \\
\hline & & & \\
\hline & & \multirow{2}{*}{\multicolumn{2}{|c|}{$\begin{array}{l}\text { 10. Work Unit No. } \\
505-62-50\end{array}$}} \\
\hline \multirow{4}{*}{\multicolumn{2}{|c|}{$\begin{array}{l}\text { 9. Performing Organization Name and Address } \\
\text { National Aeronautics and Space Administration } \\
\text { Lewis Research Center } \\
\text { Cleveland, Ohio } 44135-3191\end{array}$}} & & \\
\hline & & \multirow{2}{*}{\multicolumn{2}{|c|}{ 11. Contract or Grant No. }} \\
\hline & & & \\
\hline & & \multirow{2}{*}{\multicolumn{2}{|c|}{$\begin{array}{l}\text { 13. Type of Report and Period Covered } \\
\text { Technical Memorandum }\end{array}$}} \\
\hline \multirow{2}{*}{\multicolumn{2}{|c|}{$\begin{array}{l}\text { 12. Sponsoring Agency Name and Address } \\
\text { National Aeronautics and Space Administration } \\
\text { Washington, D.C. } 20546-0001\end{array}$}} & & \\
\hline & & \multicolumn{2}{|c|}{ 14. Sponsoring Agency Code } \\
\hline \multicolumn{4}{|l|}{ 15. Supplementary Notes } \\
\hline \multicolumn{4}{|c|}{$\begin{array}{l}\text { Prepared for the International Joint Conference on Neural Networks cosponsored by the International Neural Network } \\
\text { Society and the Institute of Electrical and Electronics Engineers, Seattle, Washington, July 8-12, 1991. T.-H. Guo, NASA } \\
\text { Lewis Research Center; J. Nurre, Ohio University, Dept. of Electrical and Computer Engineering, Athens, Ohio } 45701 \text { and } \\
\text { NASA/OAI Summer Faculty Fellow at NASA Lewis Research Center. Responsible person, T.-H. Guo, (216) 433-3734. }\end{array}$} \\
\hline \multicolumn{4}{|c|}{$\begin{array}{l}\text { 16. Abstract } \\
\text { This paper describes a new method of sensor failure detection, isolation, and accommodation using a neural network } \\
\text { approach. In a propulsion system such as the Space Shuttle Main Engine, the dynamics are usually very complicated } \\
\text { and sometimes not well known. However, the number of variables measured is usually much higher than the order of } \\
\text { the system. This built-in redundancy of the sensors can be utilized to detect and correct sensor failure problems. The } \\
\text { goal of the proposed scheme is to train a neural network to identify the sensor whose measurement is not consistent } \\
\text { with other sensor outputs. Another neural network is trained to recover the value of critical variables when their } \\
\text { measurements fail. Techniques for training the network with a limited amount of data are developed. The proposed } \\
\text { scheme is tested using the simulated data of the Space Shuttle Main Engine (SSME) inflight sensor group. }\end{array}$} \\
\hline 17. Key Words (Suggested by Author(s)) & & \multirow{2}{*}{\multicolumn{2}{|c|}{$\begin{array}{l}\text { 18. Distribution Statement } \\
\text { Unclassified - Unlimited } \\
\text { Subject Category } 63\end{array}$}} \\
\hline $\begin{array}{l}\text { Failure detection } \\
\text { Neural net } \\
\text { Artificial intelligence } \\
\text { Failure isolation }\end{array}$ &  & & \\
\hline 19. Security Classif. (of the report) & 20. Security Classif. (of this page) & 21. No. of pages & 22. Price* \\
\hline Unclassified & Unclassified & 8 & $\mathrm{~A} 02$ \\
\hline
\end{tabular}

